\title{
Le financement du système éducatif français et l'enjeu de la performance
}

Funding the French education system and the issue of performance

La financiación del sistema educativo francés y el reto de la performancia

Jean-Richard Cytermann

\section{OpenEdition}

Journals

Édition électronique

URL : http://journals.openedition.org/ries/3701

DOI : 10.4000/ries.3701

ISSN : 2261-4265

Éditeur

Centre international d'études pédagogiques

Édition imprimée

Date de publication : 15 avril 2014

Pagination : 121-131

ISBN : 978-2-85420-603-6

ISSN : $1254-4590$

\section{Référence électronique}

Jean-Richard Cytermann, « Le financement du système éducatif français et l'enjeu de la performance », Revue internationale d'éducation de Sèvres [En ligne], 65 | avril 2014, mis en ligne le 15 avril 2016, consulté le 06 janvier 2020. URL : http://journals.openedition.org/ries/3701 ; DOI : 10.4000/ries.3701 


\section{Le financement du système éducatif français et l'enjeu de la performance ${ }^{1}$}

\section{Jean-Richard Cytermann}

Lorsqu'on examine l'évolution de l'indicateur utilisé pour la comparaison internationale des dépenses d'éducation, le rapport des deux agrégats que sont la dépense intérieure d'éducation (DIE) et le produit intérieur brut (PIB), c'est une impression de stabilité qui prédomine. Le ratio DIE/PIB change peu et les traits caractéristiques du système éducatif français en matière de financement ne varient guère : sous-financement du premier degré et, relativement, de l'enseignement supérieur, sur-financement du lycée. Au-delà de cette stabilité apparente, que nous examinerons par la suite, le financement du système éducatif a connu des transformations ou des tentatives de transformation, dans le cadre de réformes institutionnelles parfois provoquées par des comparaisons internationales. Ces transformations ou tentatives de transformation ont suscité et suscitent encore des questions et des problématiques qui seront abordées à la fin de cet article.

\section{UN FINANCEMENT \\ DANS LA MOYENNE DES PAYS DÉVELOPPÉS \\ Les agrégats, seul moyen de comparer l'évolution des financements}

Lorsqu'on parle de financement de l'éducation nationale, on a tendance à se référer au concept de budget de l'éducation nationale, tel qu'il est présenté au Parlement chaque année. En fait, cette notion n'est pas le meilleur moyen d'appréhender l'effort de la collectivité en faveur de l'éducation. Il convient d'utiliser les agrégats de la comptabilité nationale mesurant la dépense d'éducation, globale ou par niveau.

En matière d'enseignement scolaire, l'État, même s'il reste de loin le plus important, n'est pas le seul financeur de la dépense d'éducation, compte tenu notamment de la part croissante des collectivités territoriales, depuis les lois de décentralisation.

1. Cet article, rédigé à titre personnel, n'engage à aucun titre ni l'inspection générale de l'administration de l'éducation nationale, ni les fonctions occupées antérieurement par l'auteur. 
En matière d'enseignement supérieur, même au niveau de l'État, les financements sont multiples et ne sont pas tous inscrits au budget du seul ministère de l'enseignement supérieur, à l'exemple des moyens des classes postbaccalauréat, qui sont inscrits au budget de l'éducation nationale. Se sont ajoutés, ces dernières années, des financements extra budgétaires, comme le plan Campus ou les investissements d'avenir.

Les aléas de l'exécution budgétaire font que les budgets exécutés sont parfois sensiblement différents des budgets prévisionnels, et que les comparaisons ne peuvent légitimement porter que sur les dépenses exécutées. Par exemple, ces dernières années se sont révélées des "insuffisances en gestion " en ce qui concerne la masse salariale des personnels ou les bourses d'enseignement supérieur, qu'il a fallu compenser.

Si l'on procède à des comparaisons internationales dans le temps et dans l'espace, c'est donc à partir des agrégats comme la dépense intérieure d'éducation (DIE) qu'il faut raisonner ${ }^{2}$. Cet agrégat intègre en effet les dépenses exécutées de la totalité des financeurs du système éducatif. Il permet de calculer des coûts à l'élève ou à l'étudiant.

\section{Stabilité de l'effort global et infléchissement en faveur de l'enseignement supérieur}

L'analyse des données produites par la Direction de l'évaluation, de la prospective et de la performance (DEPP) dans ses différentes publications et d'autres fournies par l'Organisation de coopération et de développement économique (OCDE), dans les éditions successives de Regards sur l'éducation, amène aux constatations suivantes :

- la dépense intérieure d'éducation (DIE), qui avait diminué depuis le milieu des années 1995 (7,6 \% du PIB en 1997, 7,2\% en 2002) est quasiment stable depuis 2004 (7,0 \% en 2004, 6,7 \% en 2007, 6,9\% en 2012);

- dans cette stabilité globale, la part de la DIE consacrée à l'enseignement supérieur a augmenté : elle représentait $16,6 \%$ en 2002 contre plus de $20 \%$ en 2012 ;

- en matière de comparaisons internationales, le ratio français, avec

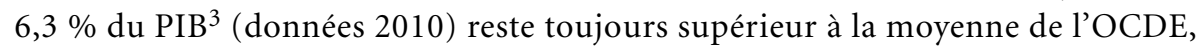
avec $5,9 \%$; ces chiffres sont identiques à ceux qui figuraient dans l'édition 2006 de Regards sur l'éducation (données 2003).

2. Voir C. Szymankiewicz (2013) « Les dépenses d’éducation ", Notice 6, in : Le système éducatifen France, $4^{\mathrm{e}}$ édition, La documentation française, 2013.

3. Le périmètre de la dépense d'éducation pour les comparaisons internationales ne comprend pas les dépenses de formation continue et incorpore un périmètre en matière de recherche à l'université plus vaste que pour le calcul national de la dépense intérieure d'éducation (DIE). 
Si l'on raisonne en termes de coût de l'élève ou de l'étudiant, la répartition de l'effort financier entre les différents ordres d'enseignement reste totalement atypique ${ }^{4}$ : la France est à la fois le pays où l'écart entre le coût d'un élève dans le primaire et le coût d'un lycéen est le plus élevé et où l'écart entre le coût d'un étudiant et celui d'un lycéen est le plus faible (l'écart de coût entre un élève du primaire et un élève du lycée est de $20 \%$ environ dans les pays de l'OCDE contre près de $90 \%$ en France). Ce choix constant en faveur du lycée au détriment à la fois de l'enseignement supérieur et de l'enseignement primaire est de plus en plus critiqué au nom de l'équité, s'agissant de l'enseignement primaire, et au nom de l'efficacité économique pour l'enseignement supérieur, dans une période où l'on considère que la qualité du système enseignement supérieur-recherche-innovation est devenue cruciale pour asseoir la compétitivité des économies développées.

Depuis 1989, le budget de l'éducation nationale, enseignement supérieur compris, est le premier budget de l'État, devant celui de la défense nationale et cette constatation renforce l'idée que la France est un pays qui dépense beaucoup pour l'éducation ${ }^{5}$. La réalité est plus nuancée : si l'on prend en compte la part des dépenses d'éducation dans les dépenses des administrations publiques (État, collectivités territoriales et organismes de sécurité sociale), le ratio français (11\%) est sensiblement inférieure à la moyenne de l'OCDE (13\%). La cause en est le poids des dépenses sociales, liées à la crise économique (exonération de charges sociales, allocations de chômage) et au vieillissement de la population (dépenses de santé et de retraites).

\section{Une absence de désengagement de la puissance publique}

Contrairement à ce qui a pu être allégué parfois, il n’y aucun désengagement de la puissance publique, ni dans l'enseignement scolaire, si l'on additionne financement de l'État et celui des collectivités territoriales, ni dans l'enseignement supérieur. Le financement public (État et collectivités territoriales) est ainsi de $83,7 \%$ en 1980 et de $85,1 \%$ en 2011 , avec une répartition différente entre État et collectivités territoriales (pour ces dernières, on est passé de $14,2 \%$ en 1980 à $25 \%$ en 2011). Le poids des entreprises et des ménages demeure réduit et a même diminué. Cette évolution se réfère à des phénomènes connus. Les droits d'inscription à l'université restent faibles, la gratuité des

4. J.-R. Cytermann, « Les choix budgétaires en matière d'éducation ", Pouvoirs n ${ }^{122}$, juillet 2007.

5. Les interprétations, sur ce point, des comparaisons internationales sont parfois abusives. La France a un effort éducatif de même ampleur, ramené à son PIB, que le Royaume-Uni et un poids des dépenses éducatives dans les dépenses publiques comparable à l'Allemagne. Ses coûts à l'élève ou à l'étudiant sont assez proches de ceux de l'Allemagne, dont l'effort éducatif moindre est dû en partie à une part plus faible de la population totale. Et les différences de statuts des personnels entre les différents pays rendent aléatoire la ventilation entre personnels enseignants et non enseignants et donc toute interprétation sur un poids excessif des non enseignants en France. 
manuels scolaires s'est étendue aux lycées à l'initiative des régions et l'investissement des entreprises dans l'éducation est peu important, si on le compare à d'autres pays européens comme l'Allemagne, avec le financement des formations par alternance.

\section{Une stabilité des moyens accordés à l'enseignement ?}

La stabilité globale de l'effort de financement, telle qu'elle apparaît au niveau macroéconomique, mais aussi la forte augmentation apparente de l'effort en faveur de l'enseignement supérieur doivent être interprétées avec précaution.

Une partie de l'augmentation des dépenses s'explique purement par des mesures techniques dites "de périmètre ». Ainsi, les cotisations pour pension versée par l'État à ses fonctionnaires ont largement augmenté sur la période. Cette augmentation est légitiment répercutée dans le calcul de la dépense d'éducation, conformément aux règles de calcul reconnues internationalement, mais elle n'apporte en elle-même aucun moyen nouveau. De même, une partie de l'augmentation de la dépense d'enseignement supérieur est due à une meilleure prise en compte des dépenses de recherche exécutées dans les universités.

Il peut y avoir contradiction entre une réelle stabilité des moyens, telle qu'elle apparaît effectivement au budget de l'État, et ce qui est ressenti sur le terrain. Prenons ainsi le cas d'une université dont les salaires représentent $80 \%$ $\mathrm{du}$ budget. Cette masse salariale peut (sous l'effet des promotions, du « glissement vieillesse-technicité ») augmenter spontanément de $1 \%$ par an. À moyens constants, les autres dépenses de fonctionnement ou d'investissement diminuent alors de $4 \%$.

Les analyses sur le financement de l'éducation ne peuvent donc se limiter aux évolutions globales des financements, telles qu'elles apparaissent dans les budgets de l'État.

\section{DES TRANSFORMATIONS STRUCTURELLES ?}

\section{La Loi organique relative aux lois de finances (LOLF)}

La Loi organique relative aux lois de finances (LOLF) votée en 2001, et appliquée à partir de 2006, a modifié considérablement les modes de présentation, de vote au Parlement et de contrôle de l'exécution des budgets tout en se voulant un mode de transformation profonde de l'action publique. Par rapport au sujet que nous traitons ici, elle a trois implications majeures.

1) Il n'y a plus de budget de l'éducation nationale stricto sensu. Les crédits correspondant à l'enseignement primaire et secondaire émargent à une 
mission interministérielle de l'enseignement scolaire, où l'on trouve également l'enseignement agricole, et ceux de l'enseignement supérieur à une mission interministérielle recherche et enseignement supérieur. Cette division en deux missions va de pair avec une séparation en deux ministères, distincts depuis 2007.

2) La logique de la LOLF est de mettre en rapport, dans les documents budgétaires, les objectifs et les résultats de l'action publique, sous forme d'indicateurs, avec les moyens de l'action publique.

3) La LOLF s'est accompagnée d'une forte globalisation des moyens et des emplois, au niveau des services déconcentrés (rectorats) pour l'enseignement scolaire, et au niveau des établissements d'enseignement supérieur pour l'enseignement supérieur. C'est certainement ce dernier point qui est le plus porteur de transformations.

\section{La politique de réforme de l'État}

La mise en œuvre de la LOLF n'est qu'un des éléments de l'introduction en France du «nouveau management public ». L'idée est, compte tenu des déficits persistants des finances publiques, de conduire en France une politique visant à réaliser des économies structurelles, à l'instar ce qui s'est fait, par exemple au Canada, sous forme de " revue » (au sens de révision) des politiques publiques. De ce point de vue, il y a, en France, une certaine continuité, au moins dans les objectifs, entre l'exercice de révision générale des politiques publiques (RGPP), menée entre 2007 et 2012, et celui de la modernisation de l'action publique (MAP), actuellement en cours.

Entre 2007 et 2012, l'éducation nationale et l'enseignement supérieur ont été traités de manière différenciée par rapport à la mesure emblématique de la révision générale des politiques publiques qu'est le non remplacement d'un départ à la retraite sur deux. Cette mesure a épargné totalement l'enseignement supérieur, mais pas l'éducation nationale.

\section{La décentralisation en matière d'éducation}

Le financement des enseignements scolaires repose en France sur un partage de compétences entre l'État et les collectivités territoriales, communes pour le primaire, départements pour les collèges et régions pour les lycées.

Le transfert aux régions et aux départements des personnels ouvriers et de service des lycées et collèges, décidé en 2004 et applicable au $1^{\text {er }}$ janvier 2006, a d'une certaine manière rapproché les situations du primaire et du secondaire, au regard des compétences respectives de l'État et des collectivités territoriales : aux collectivités territoriales le fonctionnement, l'investissement et la rémunération d'une large part des personnels non enseignants ; à l'État la rémunération des personnels enseignants. Le budget du ministère de l'éducation nationale est 
devenu donc, à plus de $90 \%$, un budget de rémunération des personnels et même des personnels enseignants.

Cette situation a pour conséquences, lorsqu'on recherche des économies dans ce secteur, à la fois de réduire massivement ce qui n'est pas directement destiné aux dépenses de rémunérations, à commencer par exemple par les crédits de formation continue des enseignants, et de toucher, par la suite, au cœur de l'action du ministère, en supprimant effectivement des emplois. Cette situation explique notamment les choix qui ont été fait, sous le gouvernement précédent, de sacrifier la politique de formation initiale des enseignants et, depuis plus longtemps, les dépenses de formation continue de tous les personnels.

\section{La réforme des universités de 2007}

La loi du 10 août 2007, intitulée « Loi relative à la liberté et à la responsabilité des universités (LRU) » a changé radicalement la gestion des universités. Elle s'est accompagnée en fait d'une véritable globalisation des moyens :

- avec le transfert de la masse salariale et des emplois correspondants, autrefois gérés directement par l'État, l'ensemble des moyens affectés aux universités a été inscrit dans leurs budgets, qui se sont retrouvés ainsi multipliés par trois ;

- les universités reçoivent une dotation globale, même si la masse salariale est distinguée à l'intérieur de celle-ci; elles peuvent arbitrer librement entre fonctionnement et investissement, entre enseignement et recherche et, dans la logique de "fongibilité asymétrique ${ }^{6}$ qui est celle de la LOLF, transférer des crédits de masse salariale disponibles vers le fonctionnement et l'investissement, mais non l'inverse ;

- à l'intérieur d'une masse salariale limitative et d'un plafond d'emploi, elles peuvent arbitrer entre nombre et niveau des emplois, requalifier les emplois ou construire des politiques indemnitaires.

\section{L’impact des comparaisons internationales sur la performance des systèmes éducatifs}

Notre système éducatif a été confronté, depuis les années 2000, à deux exercices de comparaison internationale : PISA sur les performances des élèves de 15 ans et le classement de Shanghaï des meilleures universités, qui ont amené à s'interroger sur la performance de notre système éducatif et universitaire.

6. La fongibilité asymétrique est un principe comptable établi en France par la loi organique relative aux lois de finances (LOLF), qui permet à un gestionnaire d'utiliser des crédits pour des dépenses pour lesquelles elles n'étaient pas prévues à l'intérieur d'un programme, mais sans qu'il lui soit possible d'accroître les crédits de personnel en utilisant des crédits prévus pour d'autres natures de dépenses (source : Wikipédia). (NdIR) 
Des conséquences différentes en ont été tirées en matière d'enseignement scolaire et d'enseignement supérieur.

Depuis la mise en place par l'OCDE des évaluations internationales au début des années 2000, s'est posée de manière récurrente la question de l'efficacité des dépenses d'éducation en France ${ }^{7}$. On la retrouve, de manière plus ou moins sophistiquée, dans les rapports parlementaires sur le budget de l'éducation, dans ceux de la Cour des comptes, ou dans la lettre de mission, en 2007, du président de la République au ministre de l'éducation nationale, qui constatent que, bien que le nombre d'élèves ait diminué et que le nombre d'enseignants ait augmenté, les résultats obtenus sont moyens ${ }^{8}$ et ont tendance à se dégrader. Ce rapprochement des moyens et des résultats des politiques publiques est aussi un des principes de la LOLF. Les analyses faites par l'OCDE, en comparant résultats à PISA et moyens mis dans l'éducation, rejoignent les conclusions de la plupart des économistes de l'éducation ${ }^{9}$ : à partir d'un certain niveau de développement, il n'y a plus de corrélation entre les moyens investis dans l'éducation et les résultats : ainsi, la France et la Finlande ont des dépenses d'éducation équivalentes et des résultats très différents; de même, la République tchèque et les États-Unis ont des résultats équivalents mais des niveaux d'investissements éducatifs très différents. Une note récente de l'OCDE, intitulée "Does money buy strong performance in PISA ? " conclut que "parmi les économies développées, le montant de la dépense d'éducation est moins important que la manière dont on utilise les ressources et que les systèmes scolaires les plus performants privilégient la qualité des enseignants ». Les résultats du système éducatif ne sont pas non plus corrélés à des paramètres qui mesurent l'intensité des moyens : taille des classes ou nombre d'heures reçues par les élèves. C'est ce type d'analyse qui a justifié les mesures d'économies faites sur le budget de l'éducation nationale entre 2003 et 2012.

$\mathrm{Au}$ contraire, la publication du classement de Shanghaï a été surinterprétée comme indiquant un retard manifeste de la France en matière d'enseignement supérieur et de recherche ${ }^{10}$, avec comme conséquences un retard dans la capacité d'innovation et la compétitivité de notre pays ${ }^{11}$. Le classement

7. J.-R. Cytermann, Th. Chevaillier, «Les financements de l'éducation en France et ailleurs : qui paye quoi ? Comment ? ", Revue trimestrielle de l'association française des acteurs de l'éducation, $\mathrm{n}^{\circ}$ 3, 2012.

8. Voir la phrase suivante citée en page 11 de l'introduction du rapport public thématique de la Cour des comptes intitulé Gérer les enseignants autrement : « En effet, si les enquêtes internationales montrent la diminution continue des performances $d$ système éducatif français depuis une décennie, les moyens financiers qui lui ont été alloués sur la période couverte par ces études ont, en revanche, connu une hausse constante alors que le nombre d'élèves décroissait. "

9. J.-R. Cytermann, « Les performances économiques du système éducatif français » in : « Comprendre l'économie française $»$ hors-série $\mathrm{n}^{\circ}$ 1, Problèmes économiques, 2012.

10. Si l'on raisonne en nombre d'universités classées dans Shanghaï, ou en nombre d'universités françaises dans les deux cent premières, la France se situe, suivant les années entre le $5^{\mathrm{e}}$ et le $7^{\mathrm{e}}$ rang, ce qui correspond à sa place dans la production scientifique mondiale.

11. Jean-Richard Cytermann, "Classements, indicateurs et politiques éducatives en France », Revue internationale d'éducation de Sèvres, 54, 2010, 83-92. 
de Shanghaï peut être considéré comme un des éléments déterminants de la politique qui a conduit à un traitement prioritaire du budget de l'enseignement supérieur et de la recherche au début du septennat précédent et est visiblement à l'origine des programmes des investissements d'avenir, principalement dédiés à l'enseignement supérieur et à la recherche. Dans les objectifs du programme "Initiatives d'excellence ", annexé à la loi de finances rectificative de 2010, figurait explicitement comme indicateur de la réussite du programme le fait d'avoir deux universités dans les vingt premières du classement de Shanghaï.

\section{LES PROBLÉMATIQUeS ACTUELLES EN MATIÈRE DE FINANCEMENT}

\section{Les limites du financement à la performance}

Contrairement aux attentes de ses promoteurs, la LOLF n'a pas changé les modes de construction du budget de l'État. Il existe bien, à côté des conférences budgétaires traditionnelles, des conférences de performance où sont examinés les objectifs et les indicateurs des différents programmes LOLF, mais elles n'ont aucun effet sur le montant des crédits affectés à tel ou tel programme et la direction du budget du ministère des finances continue à travailler essentiellement à partir de normes de cadrage transversales sur les économies, par nature de dépenses. Il est vrai que, selon Henri Guillaume, dans son livre Gestion publique, l'État et la performance ${ }^{12}$, aucun pays n'a vraiment réussi à établir un lien entre la performance et l'allocation des moyens. La raison en est simple: lorsqu'un objectif n'est pas atteint, il n'est pas toujours aisé d'en déterminer les causes et, par conséquent, d'en tirer ou non les conséquences en termes d'allocation des moyens.

En outre, le financement à la performance n'a cours ni dans l'enseignement scolaire ni dans la répartition opérée par le ministère entre les académies ni dans la répartition opérée par les recteurs entre les établissements scolaires. La notion de valeur ajoutée des établissements, introduite pour évaluer les performances des lycées au baccalauréat par la direction de l'évaluation et de la prospective (DEP) ${ }^{13}$ n'a eu aucune incidence sur l'allocation des moyens, de même que les tentatives de contractualisation avec les académies.

En revanche, le financement à la performance a été introduit dès 2007 dans l'allocation des moyens aux universités (système de répartition dit $\mathrm{SYMPA}^{14}$ ) avec $80 \%$ des moyens hors masse salariale répartis selon l'activité (en

12. H. Guillaume, G. Dureau, F. Silvent, Gestion publique, l'État et la performance, Presse de Sciences Po et Dalloz, 2002.

13. Devenue aujourd'hui la direction de l'évaluation, de la prospective et de la performance (DEPP).

14. SYMPA : système de répartition des moyens à la performance et à l'activité. 
fonction du nombre d'étudiants ou d'enseignants) et $20 \%$ selon la performance. Parmi les critères de performance utilisés figurent la valeur ajoutée de l'université pour sa réussite en licence, tenant compte de la composition de la population étudiante, ainsi que la notation des équipes de recherche faite par l'Agence d'évaluation de la recherche et de l'enseignement supérieur (AERES). Ce système n'a vraiment été appliqué intégralement que pendant deux années, en 2009 et 2010, années de forte croissance budgétaire. Il a vraisemblablement contribué, par ailleurs, à une certaine méfiance des communautés scientifiques vis-à-vis de l'AERES, en déportant l'activité d'évaluation vers une aide aux décideurs plutôt que vers une aide aux évalués eux-mêmes.

\section{L'importance ${ }^{15}$ des questions de répartition des moyens}

De nombreuses analyses convergent pour considérer qu'au-delà de la question des moyens globaux du système éducatif, il faut s'interroger sur la répartition de ces moyens et sur les inégalités importantes qui subsistent, entre établissements, entre académies ou entre les différentes filières de l'enseignement supérieur.

Les caractéristiques bien connues de notre système éducatif sur les disparités de financement entre les différents ordres d'enseignement persistent. Dans aucun autre pays de l'OCDE, la différence entre le coût de l'élève dans le primaire et le coût du lycéen ne sont aussi fortes et les différences entre le coût de l'étudiant, toutes filières confondues et du lycéen aussi faibles. Ces caractéristiques justifient pleinement la priorité en faveur de l'enseignement primaire affirmée par la loi de refondation de l'école ou l'augmentation réelle de la dépense d'enseignement supérieur effectuée ces dernières années.

La question de la répartition des moyens se pose également en termes d'équité. Les récents rapports de la Cour des comptes de 2010 et $2013^{16}$ critiquent sur ce point l'efficacité de la politique d'éducation prioritaire, en regrettant que le ministère de l'éducation nationale puisse dépenser deux fois plus pour le redoublement que pour l'éducation prioritaire. Au-delà de l'effet "redistributif » global des dépenses d'éducation et d'aide sociale, la recherche de l'équité peut se poser en termes de moyens supplémentaires d'enseignement pour les élèves appartenant aux publics les plus défavorisés. Telle était l'ambition au moins de la politique d'éducation prioritaire lancée en 1981, avec l'objectif « donner plus à ceux qui ont moins ». Les zones d'éducation prioritaire ont eu en moyenne un avantage d'encadrement de l'ordre de deux élèves par classe dans les écoles ou dans les collèges. Mais cet avantage dans l'encadrement des

\footnotetext{
15. Voir le rapport de la Cour des comptes « L'éducation face à l'objectif de réussite de tous les élèves et la gestion des enseignants ".

16. Voir « L'éducation nationale face à la réussite de tous les élèves ", rapport public thématique, mai 2010 et « Gérer les enseignants autrement ", rapport public thématique, mai 2013.
} 
élèves, a été annihilé par l'affectation extrêmement fréquente des enseignants les moins expérimentés dans ces zones.

En raisonnant en termes de coût salarial et non plus d'encadrement, la Cour des comptes arrive à un résultat inverse. Ce sont les lycées les plus prestigieux, même s'ils ont des classes surchargées, où le coût à l'élève est le plus élevé, dans la mesure où les enseignants les plus âgés et donc les plus rémunérés y sont affectés. Les mêmes travaux de la Cour, raisonnant en termes monétaires, concluent également que les inégalités territoriales ne sont pas prises en compte dans les dépenses d'éducation. En fait, les différences du coût à l'élève entre les régions ne tiennent pas à des politiques volontaires, mais à des phénomènes structurels : poids du rural qui conduit à des petites écoles ou à des établissements plus petits, donc plus coûteux à l'élève ; âge des enseignants plus élevé dans les régions de l'ouest, du sud et à Paris.

L'objectif de convergence et donc d'égalité de traitement entre les différentes universités a également été pris en compte dans la répartition des crédits et, actuellement, des créations d'emplois entre les universités. Des interrogations se sont également exprimées sur les mécanismes d'allocations internes aux universités, de l'équilibre entre enseignement et recherche ou entre master et licence, sachant en outre que les mécanismes de répartition ne sont pas articulés sur une connaissance des coûts des différentes filières de formation.

130 Enfin, dans les lycées comme dans les universités, est posée la question de la maîtrise de l'offre de formation (formations à petits effectifs, multiplication des options en lycée ou des spécialités en master)

\section{Les conséquences de l'autonomie budgétaire et financière des universités}

L'autonomie accordée aux universités en 2007, en leur attribuant la gestion de la masse salariale, n'a fait que rejoindre le droit commun de beaucoup d'établissements, à commencer par les organismes de recherche ou le réseau des œuvres universitaires. Les budgets de certaines universités ont une ampleur comparable à celle d'organismes de recherche moyens.

Les difficultés rencontrées dans sa mise en œuvre étaient prévisibles: elles peuvent d'une certaine manière être comparées à ce que l'on a pu constater pour certaines collectivités territoriales, après les lois de décentralisation du début des années 1980. La responsabilité est sans doute partagée entre l'État et les universités. Dans le souci politique de «vendre la LRU auprès des universités », le gouvernement a fait valoir aux établissements les marges de liberté que leur donnerait la LRU en matière de définition des primes et indemnités et de requalification des emplois et elles ont été plutôt encouragées à saturer leur plafond d'emplois. Certaines universités n'étaient pas administrativement prêtes, 
lorsqu'elles sont passées aux compétences élargies, et elles ont parfois pris des décisions imprudentes en matière de recrutement de personnels ou de régimes indemnitaires. Ces comportements expliquent en partie la situation d'universités qui ont connu deux budgets successifs en déficit.

Compte tenu des contraintes budgétaires globales, ces universités en difficulté financière sont appelées, accompagnées par le ministère de l'enseignement supérieur et de la recherche, à prendre leurs responsabilités pour établir un plan de retour à l'équilibre et à décider des mesures de rationalisation de leur offre de formation ou de gel d'emplois.

\section{Les perspectives}

Depuis 2012, les budgets de l'éducation nationale et de l'enseignement supérieur ont bénéficié d'un traitement prioritaire et ont progressé légèrement, avec des créations d'emplois substantielles, inscrites dans le tableau annexé à la loi de refondation de l'école (60000 sur cinq ans, dont 5000 pour l'enseignement supérieur). Ces créations sont destinées principalement à rétablir une formation des enseignants de qualité, à favoriser l'enseignement primaire et la réussite étudiante. Ce caractère prioritaire des budgets va devoir s'articuler avec la remise en ordre des finances publiques rendue nécessaire par le "pacte de responsabilité » annoncé par le président de la République et qui doit conduire à cinquante milliards d'euros d'économies sur les dépenses publiques. On peut également s'interroger sur les possibilités d'évolution du financement de notre système d'enseignement supérieur et sur sa capacité à développer des ressources propres. 
